\title{
Outcomes of immunosuppressive therapy in chronic hypersensitivity pneumonitis
}

\author{
Ayodeji Adegunsoye ${ }^{1}$, Justin M. Oldham², Evans R. Fernández Pérez ${ }^{3}$, \\ Mark Hamblin ${ }^{4}$, Nina Patel ${ }^{5}$, Mitchell Tener ${ }^{4}$, Deepa Bhanot ${ }^{4}$, Lacey Robinson ${ }^{5}$, \\ Sam Bullick ${ }^{2}$, Lena Chen ${ }^{1}$, Scully Hsu ${ }^{1}$, Matthew Churpek ${ }^{1}$, Donald Hedeker ${ }^{6}$, \\ Steven Montner ${ }^{7}$, Jonathan H. Chung ${ }^{7}$, Aliya N. Husain ${ }^{8}$, Imre Noth ${ }^{1}$, \\ Mary E. Strek ${ }^{1,9}$ and Rekha $\mathrm{Vij}^{1,9}$
}

Affiliations: ${ }^{1}$ Section of Pulmonary and Critical Care, Dept of Medicine, The University of Chicago, Chicago, IL, USA. ${ }^{2}$ Division of Pulmonary, Critical Care and Sleep Medicine, Dept of Medicine, University of California at Davis, Davis, CA, USA. ${ }^{3}$ Division of Pulmonary, Critical Care and Sleep Medicine, National Jewish Health, Denver, CO, USA. ${ }^{4}$ Division of Pulmonary, Critical Care and Sleep Medicine, University of Kansas Medical Center, Kansas City, KS, USA. ${ }^{5}$ Division of Pulmonary, Allergy and Critical Care Medicine, Columbia University Medical Center, New York, NY, USA. ${ }^{6}$ Dept of Public Health Sciences, The University of Chicago, Chicago, IL, USA. ${ }^{7}$ Dept of Radiology, The University of Chicago, Chicago, IL, USA. ${ }^{8}$ Dept of Pathology, The University of Chicago, Chicago, IL, USA. ${ }^{9}$ These authors contributed equally.

Correspondence: Ayodeji Adegunsoye, Section of Pulmonary and Critical Care, Dept of Medicine, The University of Chicago, 5841 S. Maryland Ave, Chicago, IL 60637, USA. E-mail: dejiduchicago.edu

ABSTRACT In chronic hypersensitivity pneumonitis (CHP), lack of improvement or declining lung function may prompt use of immunosuppressive therapy. We hypothesised that use of azathioprine or mycophenolate mofetil with prednisone reduces adverse events and lung function decline, and improves transplant-free survival.

Patients with CHP were identified. Demographic features, pulmonary function tests, incidence of treatment-emergent adverse events (TEAEs) and transplant-free survival were characterised, compared and analysed between patients stratified by immunosuppressive therapy. A multicentre comparison was performed across four independent tertiary medical centres.

Among 131 CHP patients at the University of Chicago medical centre (Chicago, IL, USA), 93 (71\%) received immunosuppressive therapy, and had worse baseline forced vital capacity (FVC) and diffusing capacity, and increased mortality compared with those who did not. Compared to patients treated with prednisone alone, TEAEs were 54\% less frequent with azathioprine therapy $(p=0.04)$ and $66 \%$ less frequent with mycophenolate mofetil $(\mathrm{p}=0.002)$. FVC decline and survival were similar between treatment groups. Analyses of datasets from four external tertiary medical centres confirmed these findings.

CHP patients who did not receive immunosuppressive therapy had better survival than those who did. Use of mycophenolate mofetil or azathioprine was associated with a decreased incidence of TEAEs, and no difference in lung function decline or survival when compared with prednisone alone. Early transition to mycophenolate mofetil or azathioprine may be an appropriate therapeutic approach in $\mathrm{CHP}$, but more studies are needed.

$@$ ERSpublications

Early transition to mycophenolate mofetil or azathioprine may be an appropriate therapeutic approach in CHP http://ow.ly/kAN130dRIX8

Cite this article as: Adegunsoye A, Oldham JM, Fernández Pérez ER, et al. Outcomes of immunosuppressive therapy in chronic hypersensitivity pneumonitis. ERJ Open Res 2017; 3: 000162017 [https://doi.org/10.1183/23120541.00016-2017].

This article has supplementary material available from openres.ersjournals.com

Received: Feb 112017 | Accepted after revision: June 232017

Support statement: This investigation was supported by a US National Institutes of Health T32 training grant (T32-HL007605). Funding information for this article has been deposited with the Crossref Funder Registry.

Conflict of interest: Disclosures can be found alongside this article at openres.ersjournals.com

Copyright $\odot$ ERS 2017. This article is open access and distributed under the terms of the Creative Commons Attribution Non-Commercial Licence 4.0. 


\section{Introduction}

Chronic hypersensitivity pneumonitis (CHP) is a fibrotic interstitial lung disease (ILD) that results from recurrent or long-standing exposure to environmental antigens and carries a 5-year survival as low as 25-30\% [1-4]. Persistent exposure to inhaled environmental antigens induces the development of inflammation and dysregulation of immune mechanisms mediated by T-lymphocytes [1]. The heterogeneous nature of these immune responses and variation in the clinical course of patients with CHP suggests that multiple pathways may be involved in disease progression. While identification of an inciting antigen has been associated with improved survival, $>50 \%$ of patients with CHP may not have an identifiable causative antigen [2]. Furthermore, despite antigen avoidance, pulmonary fibrosis may continue to progress, implying perpetuation of the underlying complex dysregulated inflammatory pathways that drive pulmonary fibrosis and increase mortality.

Progression of disease frequently leads to substantial clinical impairment and worsened quality of life, prompting the use of immunosuppressive therapy to blunt the pulmonary inflammation that perpetuates the development of fibrosis $[5,6]$. There have been no randomised, double-blind, placebo-controlled clinical trials that demonstrate the efficacy of specific therapy in improving lung function, quality of life or survival in CHP. Based on the assumed benefit, patients with CHP are widely treated with corticosteroids, which are associated with a variety of adverse events $[1,5,7,8]$. While prednisone has been the preferred immunosuppressive therapy, inhibitors of T-lymphocyte proliferation such as azathioprine or mycophenolate mofetil may be prescribed to allow tapering of prednisone [1,9]. Evidence for use of these therapies in CHP is limited. Whether their use affects the risk of adverse events and outcomes is unclear [9].

In this investigation, we conducted a retrospective longitudinal analysis of patients with CHP to determine whether the use of azathioprine or mycophenolate mofetil with prednisone was associated with a decreased incidence of adverse events, reduced the decline in \% predicted forced vital capacity (FVC) and improved transplant-free survival. Patients receiving only prednisone were used as a control group, as this is the most widely prescribed therapy in patients with CHP $[5,10]$. We then analysed datasets obtained from four tertiary ILD centres (National Jewish Health, Denver, CO, USA; University of Kansas Medical Center, Kansas City, KS, USA; Columbia University Medical Center, New York, NY, USA; and University of California at Davis Medical Center, Davis, CA, USA) to determine whether their data supported our findings.

\section{Methods}

This investigation was conducted at the University of Chicago (Chicago, IL, USA) and approved by our Institutional Review Board (IRB) (protocol number 14163-A). We identified patients in the University of Chicago ILD registry followed from 2006 to 2015 with a multidisciplinary diagnosis of CHP. A multidisciplinary diagnosis of $\mathrm{CHP}$ is performed in a rigorous fashion in conjunction with pulmonologists, dedicated chest radiologists and thoracic pathologists. Two chest radiologists (J.H. Chung and S. Montner) reviewed high-resolution computed tomography (HRCT) of patients with CHP, and characterised and scored them for features of fibrosis [11] (defined as the presence of reticulation, traction bronchiectasis and honeycombing). A systematic, detailed historical assessment of environmental antigen exposures (avian, mould, hot tub and unknown) is performed for all patients in the University of Chicago ILD registry, regardless of referring diagnosis. The presence of antibodies to serum precipitins supported the diagnosis but was not a requirement. The electronic medical record was reviewed to identify patients within this group treated with prednisone, azathioprine or mycophenolate mofetil. Therapy with azathioprine or mycophenolate mofetil occurred in addition to prednisone. We extracted other pertinent data from the electronic medical record including demographic information (age, race/ethnicity and sex), tobacco use, medication doses and duration, physical examination findings including clubbing and crackles, pulmonary function testing (PFT) including \% predicted FVC and \% predicted diffusing capacity of the lung for carbon monoxide (DLCO), and diagnostic studies (HRCT and surgical lung biopsies).

We compared demographic characteristics and PFTs between patients who received immunosuppressive therapy and those who did not. We then compared these characteristics between subgroups of patients stratified by type of immunosuppressive therapy. The first period of treatment with immunosuppressive therapy after establishing care at our institution was used to conduct this analysis. Crossing over from azathioprine to mycophenolate mofetil, or mycophenolate mofetil to azathioprine, was allowed if it occurred within 4 weeks of therapy initiation and was due to a nonrespiratory side-effect. Adverse events were evaluated from baseline until 28 days after the last dose of immunosuppressive therapy or study termination. Treatment-emergent adverse events (TEAEs) were defined as adverse events documented in the drug safety labelling, not present at baseline, that occurred after immunosuppressive therapy use. We determined the incidence of TEAEs and classified by type of immunosuppressive therapy. TEAEs were graded according to the National Institutes of Health/National Cancer Institute Common Terminology 
Criteria for Adverse Events (CTCAE) [12]. Serious adverse events (SAEs) were defined as death, lung transplantation and respiratory hospitalisation. The electronic medical record, social security death index and telephone communication with patients and family members were used to ascertain adverse events. Follow-up time was censored on December 1, 2015. Patients with at least two PFTs $\geqslant 90$ days apart were included in the longitudinal PFT analysis.

\section{External validation}

Datasets of patients with CHP were also obtained from four independent tertiary ILD centres (National Jewish Health Hospitals (IRB number 1603), University of Kansas Medical Center (IRB number 13640), Columbia University Medical Center (IRB number AAAN7911) and University of California at Davis Medical Center (IRB number 585448)) and analysed. These datasets were stratified by type of immunosuppressive therapy received, and analysed for FVC change and transplant-free survival.

\section{Statistical analysis}

Continuous variables are presented as mean \pm SD or median (interquartile range) and were compared using a two-tailed Student's t-test. Categorical variables are presented as $\mathrm{n}(\%)$ and compared using the Chi-square test or Fisher's exact test, as appropriate. Adverse events, including death, lung transplantation and respiratory hospitalisation, are presented as $\mathrm{n}$ with multiple events possible for a given patient. A combined end-point incidence was determined for each treatment subgroup. The incidence rate ratio (IRR) for each treatment subgroup was assessed using negative binomial regression.

Longitudinal analysis of pulmonary function change associated with prednisone and mycophenolate mofetil therapy was conducted using mixed-effects regression models. Based on exploratory analysis with restricted maximum likelihood modelling, an autoregressive structure was chosen for FVC modelling. PFTs were grouped into 90-day intervals to allow for time course alignment. To enable evaluation of the composite effect of non-corticosteroid immunosuppressive therapy, we constructed a new disease-modifying drug (DMD) subgroup (composed of individuals receiving either azathioprine or mycophenolate mofetil). Survival time was defined as the time from diagnosis to death, transplant, loss to follow-up or the end of the study period. Survival time was censored on December 31, 2015, or at the time a patient underwent lung transplant or was lost to follow-up. Survival analysis was performed using Cox proportional hazards and depicted using Kaplan-Meier survival curves. Summary statistics with $\mathrm{p}<0.05$ were considered to be statistically significant. All statistical analyses were performed using Stata (release 14; StataCorp, College Station, TX, USA).

\section{Results}

Of 1205 patients screened from the University of Chicago ILD registry, 131 had a multidisciplinary diagnosis of CHP. All CHP patients had radiographic or pathological evidence of pulmonary fibrosis (figure 1). Of these 131 patients, 93 (71\%) were treated with immunosuppressive therapy (table 1). Patients receiving immunosuppressive therapy, as compared to those who did not, had worse baseline FVC $(60 \%$ versus $73 \%$ predicted, $\mathrm{p}<0.001)$ and DLCO $(47 \%$ versus $69 \%$ predicted, $\mathrm{p}<0.001)$, were more likely to require supplemental oxygen $(71 \%$ versus $24 \%, \mathrm{p}<0.001)$, and had higher radiographic pulmonary fibrosis score (126 versus $118, \mathrm{p}=0.01$ ).

Of the 93 patients who received immunosuppressive therapy, 41 were treated with prednisone only, 24 were also treated with azathioprine and 28 with mycophenolate mofetil. Within 4 weeks of initiating therapy, five patients in the azathioprine subgroup experienced non-respiratory side-effects and were transitioned to mycophenolate mofetil, while one patient in the mycophenolate mofetil subgroup experienced early gastrointestinal side-effects and was transitioned to azathioprine, leaving 20 treated with azathioprine and 32 treated with mycophenolate mofetil for the outcome analysis (figure 1). The reason for early-onset side-effects in these patients was unclear as all patients initiated on azathioprine therapy underwent thiopurine methyltransferase enzyme activity testing and were found to have normal enzyme activity levels. Of the immunosuppressive therapy group, 20 treated with prednisone and 20 treated with mycophenolate mofetil had multiple PFTs and were included in the longitudinal analysis of pulmonary function. Only 12 patients treated with azathioprine had multiple PFTs. This limited sample size precluded longitudinal analysis of pulmonary function in the azathioprine subgroup.

Comparing the prednisone, azathioprine and mycophenolate mofetil subgroups (table S1), the azathioprine subgroup was younger (mean \pm SD age $62 \pm 13$ versus $56 \pm 14$ versus $63 \pm 8$ years, $\mathrm{p}=0.08$ ), had more radiographic ground-glass opacities ( $85 \%$ versus $100 \%$ versus $97 \%, p=0.06)$ and was most likely to have surgical lung biopsies ( $56 \%$ versus $85 \%$ versus $69 \%, \mathrm{p}=0.08$ ). Otherwise, all three subgroups had similar baseline characteristics. The median daily dose of prednisone for patients in the prednisone subgroup was $40 \mathrm{mg}$ and the median duration of continuous prednisone therapy was 11 weeks (table S2). 


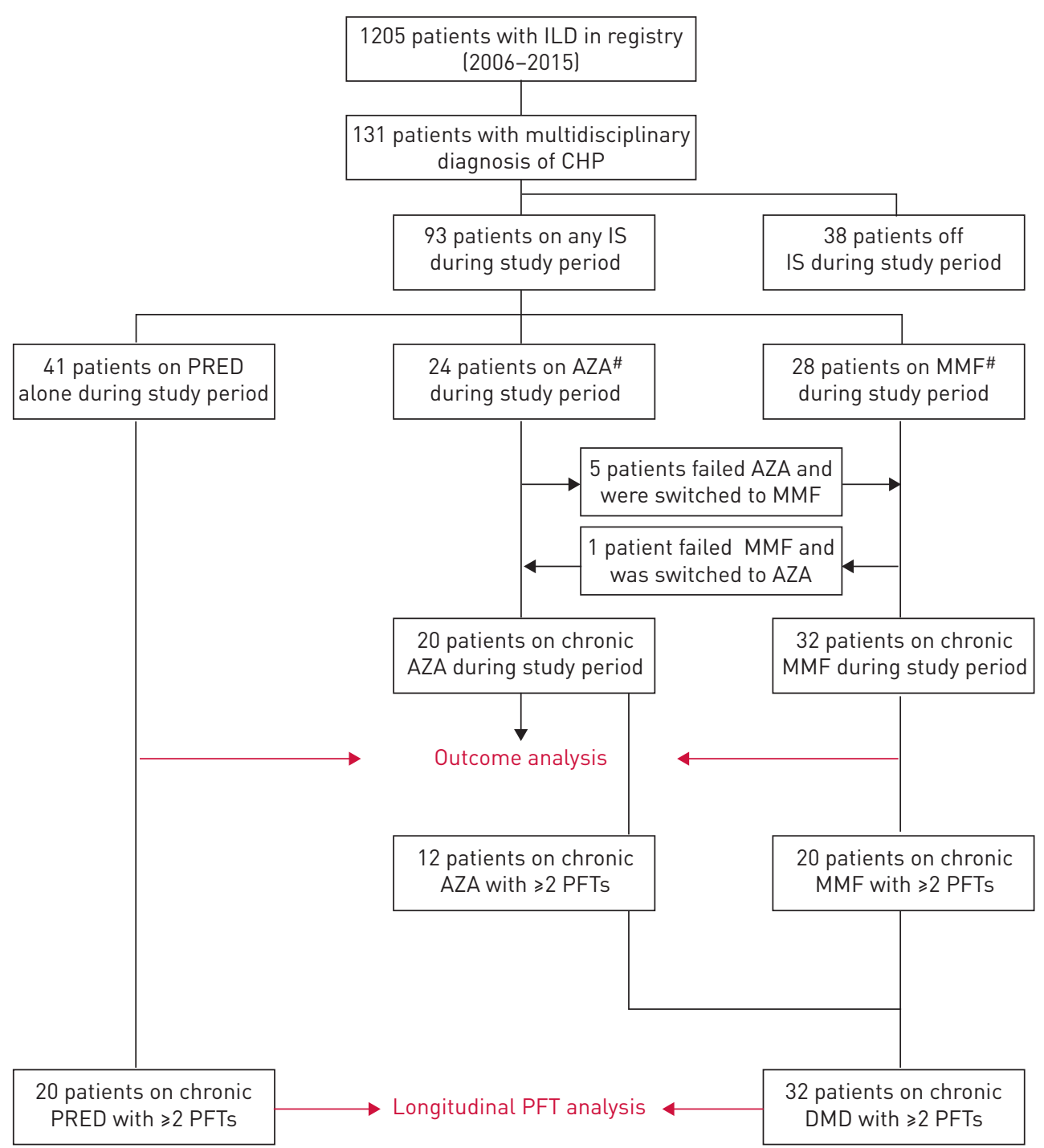

FIGURE 1 Consolidated Standards of Reporting Trials diagram. ILD: interstitial lung disease; CHP: chronic hypersensitivity pneumonitis; IS: immunosuppressive therapy; PRED: prednisone; AZA: azathioprine; MMF: mycophenolate mofetil; PFT: pulmonary function test; DMD: disease-modifying drug. " : patients receiving AZA or MMF also received PRED during the study period.

The median daily dosages of azathioprine and mycophenolate mofetil were $125 \mathrm{mg}$ and $2000 \mathrm{mg}$, respectively. For patients in the azathioprine and mycophenolate mofetil subgroups, the median daily dosages of additional therapy with prednisone were $40 \mathrm{mg}$ and $20 \mathrm{mg}$, respectively; the median durations of this additional therapy with continuous prednisone use were 8 weeks and 9 weeks, respectively (table S2).

The overall incidence of adverse events was greatest in the prednisone subgroup and least in the mycophenolate mofetil subgroup (figure S1). When comparing TEAEs between subgroups (tables 2 and S3), there were 104 adverse events over 525.4 exposure months in the prednisone subgroup (incidence rate 0.198), 30 adverse events over 401.8 exposure months in the mycophenolate mofetil subgroup (incidence rate 0.075 ) and 45 adverse events over 505.8 exposure months in the azathioprine subgroup (incidence rate 0.089). Using the prednisone subgroup as the reference, treatment with mycophenolate mofetil was associated with an IRR of 0.30 (95\% CI $0.15-0.58, \mathrm{p}<0.001)$ in the unadjusted analysis and 0.34 (95\% CI 0.17-0.67, $\mathrm{p}=0.002$ ) after adjustment for age, sex, race, FVC (\% predicted), DLCO (\% predicted) and identified antigen. Similarly, in reference to the prednisone subgroup, azathioprine therapy was associated with an IRR of 0.48 (95\% CI $0.23-0.97, \mathrm{p}=0.040)$ in the unadjusted analysis and 0.46 (95\% CI $0.21-0.98$, $\mathrm{p}=0.044$ ) in the adjusted analysis using the multivariable model described above. When adverse events were graded based on the CTCAE [13] and subcategorised by type of immunosuppressive therapy, the prednisone subgroup consistently had the highest incidence of TEAEs while the mycophenolate mofetil 


\begin{tabular}{|c|c|c|c|}
\hline Characteristics of CHP cohort $^{\#}$ & No IS ${ }^{\pi}$ & $\mathrm{IS}^{+}$ & p-value \\
\hline Age years & $65.1 \pm 10$ & $61.1 \pm 12$ & 0.07 \\
\hline Male & $19(50 \%)$ & $34(37 \%)$ & 0.16 \\
\hline Caucasian race/ethnicity & $31(82 \%)$ & $80(86 \%)$ & 0.52 \\
\hline$B M I \mathrm{~kg} \cdot \mathrm{m}^{-2}$ & $33.0 \pm 9$ & $32.6 \pm 9$ & 0.83 \\
\hline Ever-smoker & $20(53 \%)$ & $54(58 \%)$ & 0.57 \\
\hline \multicolumn{4}{|l|}{ Antigen exposure $e^{\S}$} \\
\hline Avian & $19(50 \%)$ & $42(45 \%)$ & 0.61 \\
\hline Mould & $11(29 \%)$ & $28(30 \%)$ & 0.90 \\
\hline Hot tub & $1(3 \%)$ & $2(2 \%)$ & 0.99 \\
\hline Unknown & $11(29 \%)$ & $29(31 \%)$ & 0.80 \\
\hline Gastro-oesophageal reflux & $20(53 \%)$ & $58(62 \%)$ & 0.30 \\
\hline Crackles & $28(73 \%)$ & $85(91 \%)$ & 0.008 \\
\hline Clubbing & $3(8 \%)$ & $26(28 \%)$ & 0.012 \\
\hline TLC $\%$ predicted & $79.7 \pm 19$ & $69.0 \pm 17$ & 0.002 \\
\hline FVC $^{\# \# \% \text { predicted }}$ & $73.3 \pm 19$ & $60.0 \pm 17$ & $<0.001$ \\
\hline D८coๆๆ \% predicted & $69.2 \pm 23$ & $47.2 \pm 22$ & $<0.001$ \\
\hline Oxygen therapy & $9(24 \%)$ & $66(71 \%)$ & $<0.001$ \\
\hline ANA seropositivity & $11(31 \%)$ & $26(29 \%)$ & 0.78 \\
\hline \multicolumn{4}{|l|}{ HRCT features } \\
\hline HRCT fibrosis score & $117.8 \pm 10$ & $125.9 \pm 18$ & 0.01 \\
\hline Mosaic attenuation & $31(82 \%)$ & $81(87 \%)$ & 0.42 \\
\hline Ground-glass opacities & $33(87 \%)$ & $86(93 \%)$ & 0.31 \\
\hline Honeycombing & $14(37 \%)$ & $42(45 \%)$ & 0.38 \\
\hline \multicolumn{4}{|l|}{ Histopathological features } \\
\hline SLB obtained & $18(47 \%)$ & $62(67 \%)$ & 0.04 \\
\hline Poorly formed granulomas & $11(61 \%)$ & $41(66 \%)$ & 0.69 \\
\hline Honeycombing with UIP pattern & $7(39 \%)$ & $29(47 \%)$ & 0.55 \\
\hline Follow-up time months & $43 \pm 30$ & $38 \pm 27$ & 0.37 \\
\hline \multicolumn{4}{|c|}{ 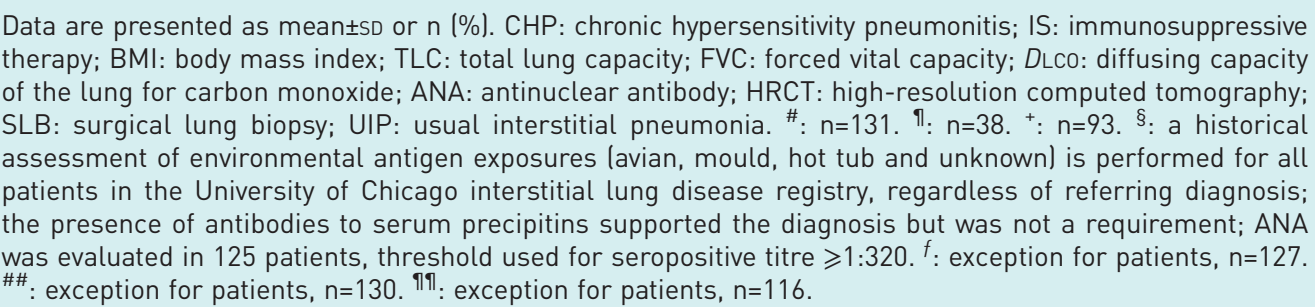 } \\
\hline
\end{tabular}

subgroup had the lowest (figure 2a). These findings were consistent when the cumulative incidence of TEAEs was evaluated over time (figure $2 b$ ).

When comparing SAEs between subgroups (table 2), there were eight SAEs over 525.4 exposure months in the prednisone subgroup (incidence rate 0.015), eight SAEs over 401.8 exposure months in the mycophenolate mofetil subgroup (incidence rate 0.020) and eight SAEs over 505.8 exposure months in the azathioprine subgroup (incidence rate 0.016). Using the prednisone subgroup as the reference, unadjusted analysis of the IRR showed no differences in SAEs with mycophenolate mofetil therapy $(1.19,95 \%$ CI $0.38-3.78 ; \mathrm{p}=0.767)$ or azathioprine therapy $(1.40,95 \%$ CI $0.27-7.33, \mathrm{p}=0.689)$; this remained consistent after adjusted the analysis using the multivariable model described earlier for mycophenolate mofetil therapy $(1.22,95 \%$ CI $0.35-4.33$; $\mathrm{p}=0.755)$ and azathioprine therapy $(0.84,95 \%$ CI 0.18-3.97; $\mathrm{p}=0.829)$.

Treatment-associated longitudinal change in \% predicted FVC is presented graphically using locally weighted scatterplot smoothing in figure S2. Patients receiving immunosuppressive therapy with prednisone, as compared to those who did not, had worse FVC decline over 36 months $(-10.0 \%$ versus $-1.3 \%, \mathrm{p}=0.042$ ) (table 3). The FVC decline over 36 months in the prednisone subgroup was similar to that of the mycophenolate mofetil subgroup (mean \pm SE change in FVC $-10.8 \pm 2.7 \%$ versus $-10.1 \pm 2.7 \%$, $\mathrm{p}=0.864$ ) (table 3). Variation in duration of therapy and the number of patients in the prednisone subgroup limited our ability to perform a subgroup analysis for a dose-response effect of prednisone on longitudinal FVC. We combined the mycophenolate mofetil and azathioprine subgroups into a DMD 
TABLE 2 Adverse events in patients with chronic hypersensitivity pneumonitis receiving immunosuppression

\begin{tabular}{|c|c|c|c|c|c|c|c|}
\hline \multirow[t]{2}{*}{ Treatment $^{\#}$} & \multirow[t]{2}{*}{ Events } & \multirow[t]{2}{*}{ Exposure months } & \multirow[t]{2}{*}{ Incidence } & \multicolumn{2}{|c|}{ Unadjusted } & \multicolumn{2}{|c|}{ Adjusted" } \\
\hline & & & & IRR $(95 \% \mathrm{CI})$ & p-value ${ }^{+}$ & IRR (95\% CI) & p-value ${ }^{+}$ \\
\hline \multicolumn{8}{|l|}{ TEAEs $^{\S}$} \\
\hline MMF (n=32) & 30 & 401.83 & 0.075 & $0.30(0.15-0.58)$ & $<0.001$ & $0.34(0.17-0.67)$ & 0.002 \\
\hline AZA $(n=20)$ & 45 & 505.83 & 0.089 & $0.48(0.23-0.97)$ & 0.040 & $0.46(0.21-0.98)$ & 0.044 \\
\hline \multicolumn{8}{|l|}{ SAEs ${ }^{f}$} \\
\hline$A Z A(n=20)$ & 8 & 505.83 & 0.016 & $1.40(0.27-7.33)$ & 0.689 & $0.84(0.18-3.97)$ & 0.829 \\
\hline
\end{tabular}

IRR: incidence rate ratio; TEAE: treatment-emergent adverse event; PRED: prednisone; MMF: mycophenolate mofetil; AZA: azathioprine; SAE: serious adverse event. " : patients receiving MMF or AZA also had concurrent therapy with low-dose PRED. ": for age, sex, race, forced vital capacity (\% predicted), diffusing capacity of the lung for carbon monoxide (\% predicted) and identified antigen. ${ }^{+}$: versus PRED. ${ }^{\S}$ : MMF TEAEs were constipation, nausea, vomiting, headache, dizziness, blurry vision, diarrhoea, stomach upset/pain, flatulence/bloating/dyspepsia, oedema, hypertension, fever, tremors, insomnia, petechiae/bruising, cellulitis, recurrent urinary tract infections and lower respiratory tract infections; PRED TEAEs were appetite changes, nausea, vomiting, hypokalaemia, headache, dizziness, blurry vision, glucose intolerance, stomach upset/ pain, flatulence/dyspepsia, oedema, sodium retention, hypertension, fever, diaphoresis, tremors, insomnia, petechiae/bruising, cellulitis, skin atrophy, impaired wound healing, facial erythema, skin pigmentation, hair loss, acne, rash, urticaria, emotional lability, anxiety, depression, raised intraocular pressure, Cushing's syndrome/moon facies, hirsutism, menstrual irregularities, muscle atrophy/deconditioning/proximal myopathy, osteopenia/osteoporosis, cataracts, glaucoma, thrush, recurrent urinary tract infections and lower respiratory tract infections. $f$ : death, lung transplant or respiratory hospitalisation.

category. In patients previously receiving prednisone and subsequently commenced on mycophenolate mofetil or azathioprine, DMD therapy significantly altered the slope of monthly FVC decline $(-0.7 \%$ versus $-0.2 \%, \mathrm{p}=0.001$ ) such that the overall $\mathrm{FVC}$ decline over 36 months in the DMD subgroup remained similar to that of the prednisone subgroup $(-9.4 \pm 4.32 \%$ versus $-11.5 \pm 3.60 \%, \mathrm{p}=0.585)$ (table 3 and figure 3). These findings remained consistent across multiple models used in the analysis for comparison (table 3 and figure S2-S4).

When evaluating survival in CHP patients, receiving immunosuppressive therapy was associated with increased mortality risk on univariate Cox regression analysis (hazard ratio (HR) 4.95, 95\% CI 1.51-16.20; $\mathrm{p}<0.01$ ) (figure $4 \mathrm{a}$ ), and remained consistent after adjustment for age, sex, race, FVC (\% predicted), DLCO (\% predicted) and identified antigen (HR 5.37, 95\% CI 1.08-26.67; $\mathrm{p}=0.04$ ). There were no differences in transplant-free survival among patients receiving prednisone alone, patients receiving azathioprine and
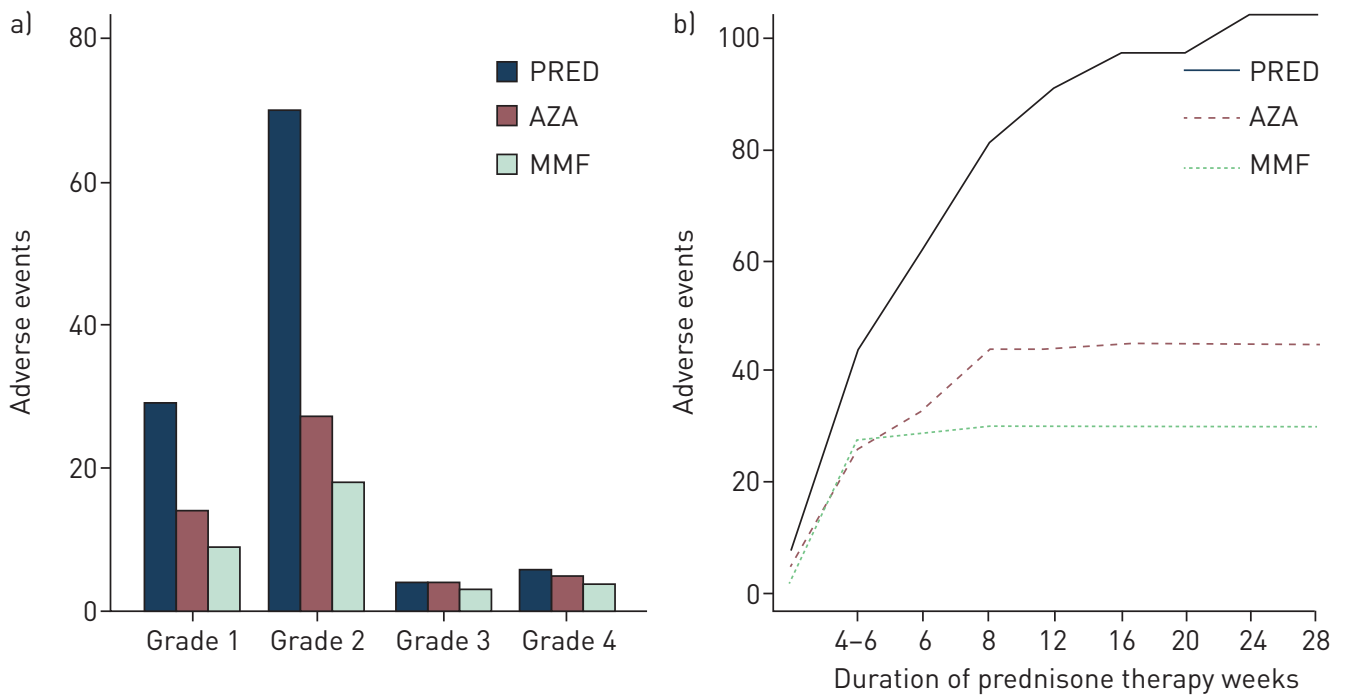

FIGURE 2 Treatment-emergent adverse events in chronic hypersensitivity pneumonitis patients receiving immunosuppressive therapy. a) Grades of adverse events based on the Common Terminology Criteria for Adverse Events and subcategorised by type of immunosuppressive therapy administered. b) Cumulative incidence of adverse events by duration of high-dose prednisone (PRED) therapy ( $\geqslant 40 \mathrm{mg} \cdot \mathrm{day}{ }^{-1}$ ). AZA: azathioprine; MMF: mycophenolate mofetil. 
TABLE 3 Model comparison for effect of additional therapy on forced vital capacity over 36 months in patients with chronic hypersensitivity pneumonitis

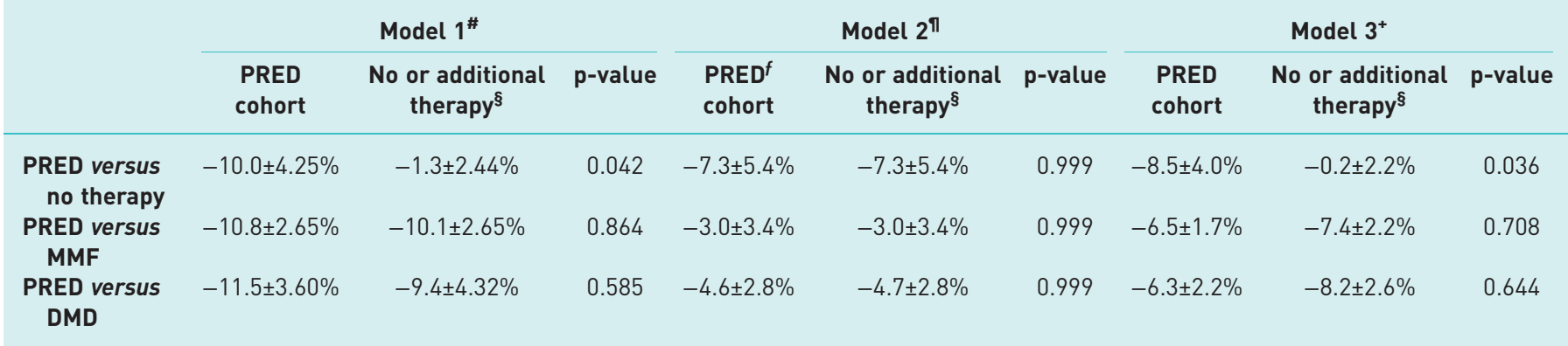

Data are presented as mean \pm SE change in forced vital capacity unless otherwise stated. PRED: prednisone; MMF: mycophenolate mofetil: DMD: disease-modifying drug (MMF or azathioprine). " : mixed-effects regression model estimates of change at baseline and at 36 weeks post-therapy; ${ }^{\text {I: }}$ difference-in-difference model estimates of change in slope before and after initiating steroid-sparing agent during the 36-week period; ${ }^{+}$: shared parameter model jointly estimates of change at baseline and at 36 weeks post-therapy in addition to survival analysis in study cohort; $;{ }^{\S}$ : additional therapy with steroid-sparing agent; ${ }^{f}$ : initiation of PRED monotherapy was associated with a mean forced vital capacity change of $-7.3 \%$.

patients receiving mycophenolate mofetil $(\mathrm{p}=0.542)$ (figure $4 \mathrm{~b}$ ). These findings remained consistent after adjustment for age, sex, race, FVC (\% predicted), DLCO (\% predicted) and identified antigen (table S4).

\section{External validation}

From the four external ILD centres, $184 \mathrm{CHP}$ patients received immunosuppressive therapy and were analysed as a secondary cohort. 113 were treated with prednisone only; 33 were also treated with azathioprine and 38 with mycophenolate mofetil (table S5). Consistent with findings in the primary University of Chicago cohort, patients in the azathioprine subgroup were younger at most external ILD centres (one centre had no patient receiving azathioprine), and the proportion of patients with an identified antigen across the prednisone, azathioprine and mycophenolate mofetil subgroups did not differ (table S5). Due to the heterogeneity of clinical data acquisition across centres, the incidence of TEAEs could not be assessed in the secondary cohort.

As observed in the primary cohort, the baseline FVC for patients in the prednisone subgroup was not significantly different from that of the azathioprine or mycophenolate mofetil subgroups at three external centres. Likewise, patients with FVC data available at 52 weeks demonstrated no significant differences in the mean FVC change across the prednisone, azathioprine and mycophenolate mofetil subgroups (table 4).

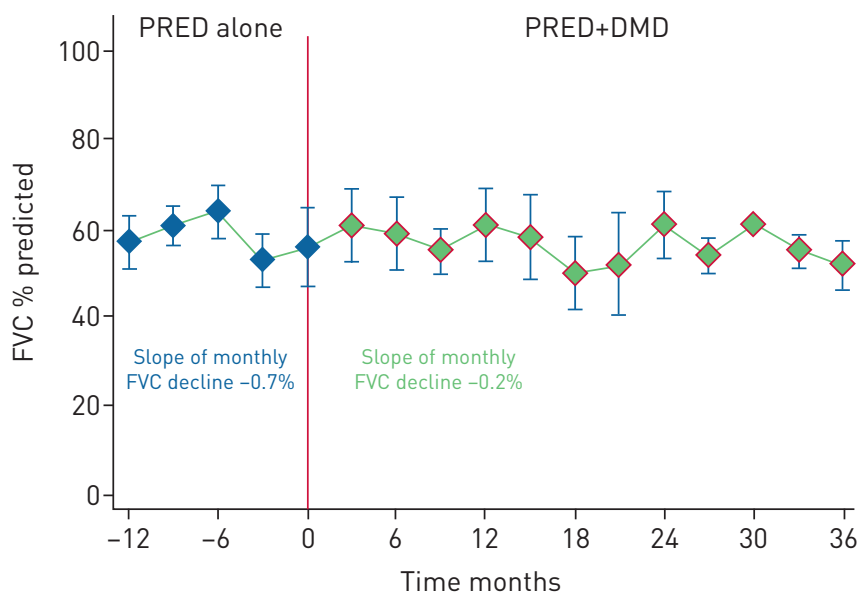

FIGURE 3 Trend of forced vital capacity (FVC) in the disease-modifying drug (DMD) subgroup by therapy administered. Within the DMD subgroup of chronic hypersensitivity pneumonitis patients who had previously received prednisone (PRED), administration of mycophenolate mofetil or azathioprine therapy significantly altered the slope of monthly FVC decline $(-0.7 \%$ versus $-0.2 \%, p=0.001)$. Data analysed using mixed regression model. 


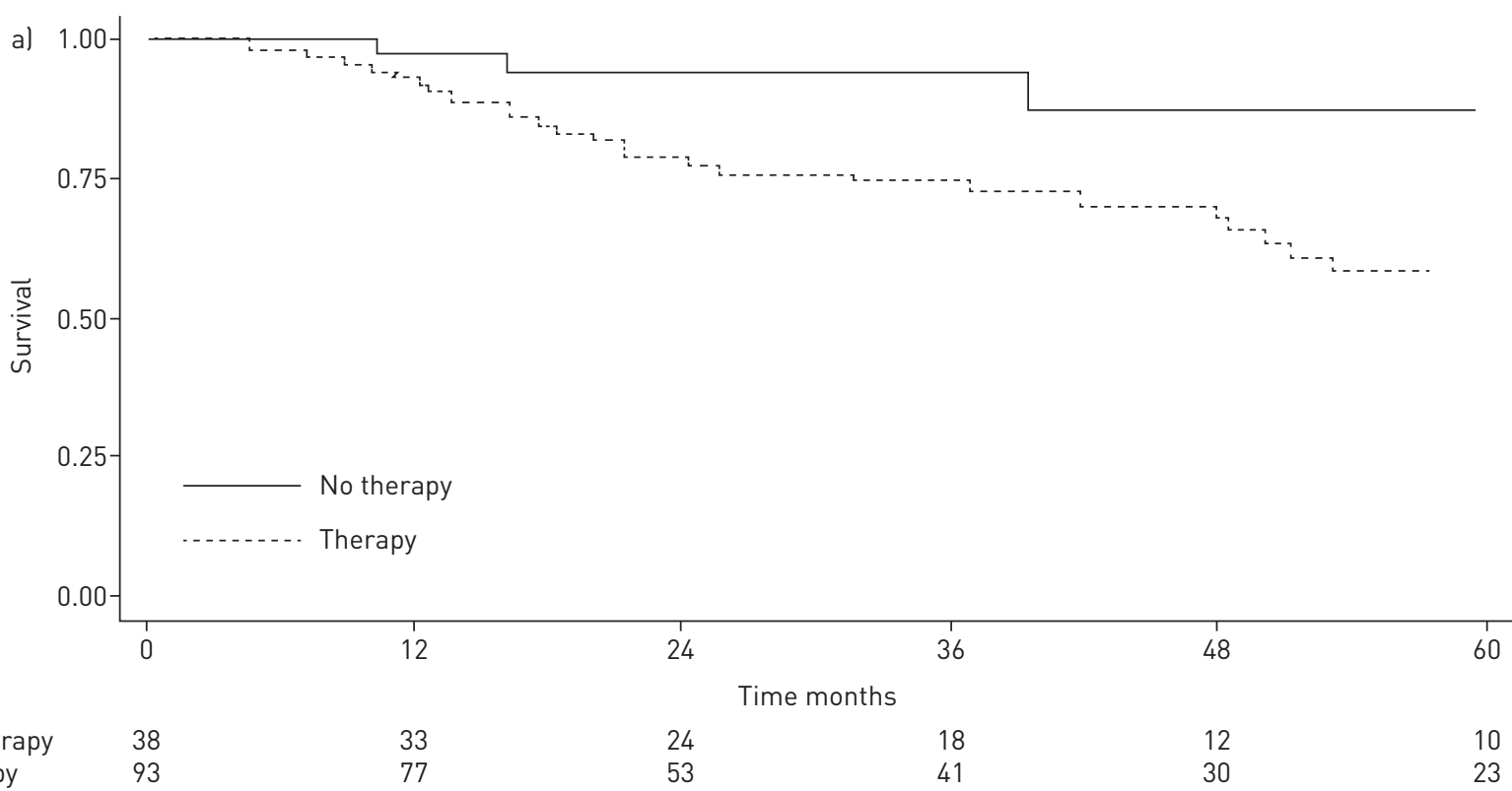

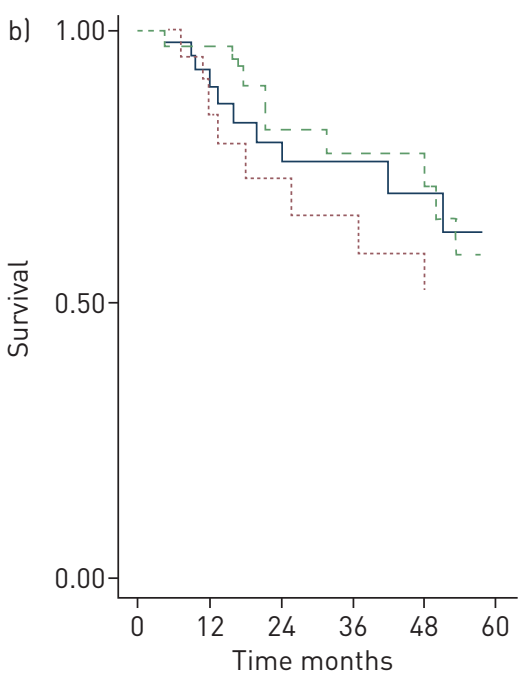

\begin{tabular}{|c|c|c|c|c|c|}
\hline PRED & 41 & 30 & 22 & 15 & 10 \\
\hline AZA & 20 & 17 & 11 & 9 & 7 \\
\hline MMF & 32 & 30 & 20 & 17 & 13 \\
\hline
\end{tabular}

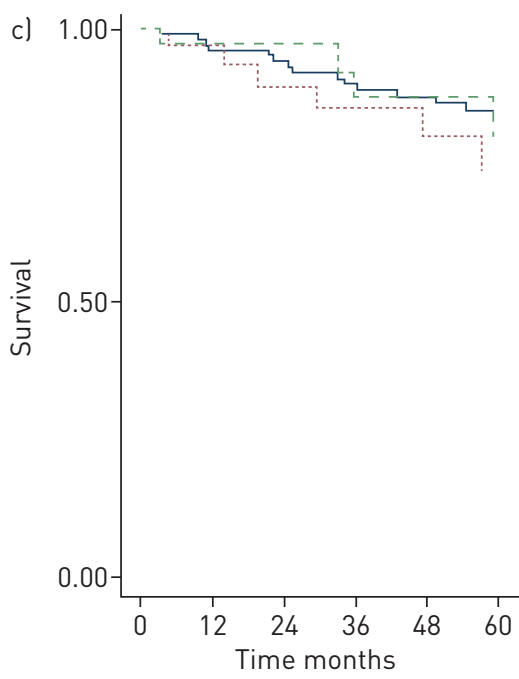

\begin{tabular}{|c|c|c|c|c|c|}
\hline PRED & 113 & 100 & 91 & 80 & 73 \\
\hline AZA & 33 & 29 & 22 & 21 & 14 \\
\hline MMF & 38 & 32 & 26 & 18 & 14 \\
\hline
\end{tabular}

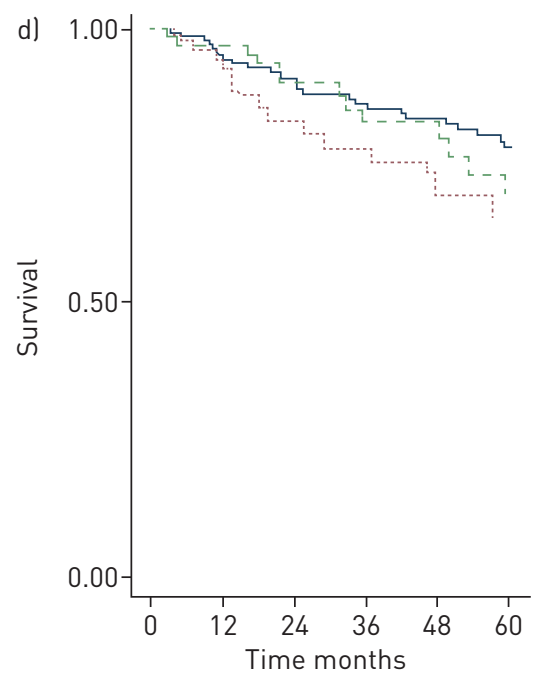

\begin{tabular}{|c|c|c|c|c|c|}
\hline PRED & 154 & 130 & 113 & 95 & 8 \\
\hline AZA & 53 & 46 & 33 & 30 & 2 \\
\hline MMF & 70 & 62 & 46 & 35 & 2 \\
\hline
\end{tabular}

FIGURE 4 Survival in chronic hypersensitivity pneumonitis (CHP) patients receiving immunosuppressive therapy. a) 5-year survival based on use of immunosuppressive therapy in the University of Chicago CHP cohort (hazard ratio (HR) 4.95, 95\% Cl 1.51-16.20; $\mathrm{p}<0.01$ ); multivariate analysis adjusting for age, sex, race, forced vital capacity (\% predicted), diffusing capacity of the lung for carbon monoxide (\% predicted) and identified antigen (HR 5.37, 95\% Cl 1.08-26.67; p=0.04). b) 5-year survival substratified by type of immunosuppressive therapy in the University of Chicago CHP cohort. c) 5-year survival substratified by type of immunosuppressive therapy in the non-University of Chicago CHP cohort. d) 5-year survival substratified by type of immunosuppressive therapy in the combined CHP cohort. PRED: prednisone; AZA: azathioprine; MMF: mycophenolate mofetil.

Similar to the findings in the primary cohort, the secondary cohort had no differences in transplant-free survival when stratified according to their prednisone, azathioprine and mycophenolate mofetil subgroups $(\mathrm{p}=0.220$ ) (figure $4 \mathrm{c}$ ). These findings remained consistent when survival was evaluated in the combined CHP cohort on immunosuppressive therapy across all centres $(n=277)(p=0.302)$ (figure $4 d)$.

\section{Discussion}

We have shown that in patients with $\mathrm{CHP}$, the requirement for immunosuppressive therapy was associated with increased mortality. Our study also showed that in CHP patients treated with prednisone, additional 


\begin{tabular}{|c|c|c|c|c|}
\hline & PRED & AZA & MMF & p-value \\
\hline \multicolumn{5}{|l|}{ Baseline FVC \% predicted } \\
\hline University of Chicago (n=93) & $61.4 \pm 19.3$ & $56.7 \pm 14.6$ & $60.2 \pm 16.3$ & 0.627 \\
\hline National Jewish Health ( $\mathrm{n}=99$ ) & $64.6 \pm 19.1$ & $66.4 \pm 21.0$ & $69.1 \pm 19.3$ & 0.730 \\
\hline University of Kansas Medical Center $(n=60)$ & $68.8 \pm 13.5$ & $57.0 \pm 14.5$ & $65.4 \pm 20.5$ & 0.093 \\
\hline Columbia University Medical Center ( $n=15$ ) & $75.0 \pm 14.0$ & & $60.0 \pm 14.0$ & 0.063 \\
\hline University of California Davis ( $n=10$ ) & $65.0 \pm 8.0$ & $34.0 \pm 0.0$ & $65.0 \pm 21.0$ & 0.278 \\
\hline \multicolumn{5}{|l|}{ Mean FVC change after 52 weeks } \\
\hline University of Chicago (n=34) & $-1.2 \pm 14.9 \%$ & $-7.6 \pm 14.8 \%$ & $-2.3 \pm 10.9 \%$ & 0.545 \\
\hline University of Kansas Medical Center $(n=60)$ & $0.7 \pm 15.5 \%$ & $5.7 \pm 11.1 \%$ & $8.8 \pm 14.6 \%$ & 0.434 \\
\hline Columbia University Medical Center ( $n=15$ ) & $-3.2 \pm 12.7 \%$ & & $-3.3 \pm 14.1 \%$ & 0.990 \\
\hline \multicolumn{5}{|c|}{$\begin{array}{l}\text { Data are presented as mean } \pm \text { SD unless otherwise stated. Due to variation in data availability across } \\
\text { multiple tertiary centres, only patients with FVC data available at baseline and at the } 52 \text {-week time-point } \\
\text { were included in this analysis; } 52 \text { week follow-up FVC data were not available for the National Jewish } \\
\text { Health and University of California Davis cohorts. National Jewish Health: prednisone (PRED), } n=73 \text {; } \\
\text { azathioprine (AZA), } n=19 ; \text { mycophenolate mofetil (MMF), } n=7 \text {. University of Kansas Medical Center: PRED, } \\
n=30 ; A Z A, n=13 ; M M F, n=17 \text {. Columbia University Medical Center: PRED, } n=6 ; A Z A, n=0 ; \text { MMF, } n=9 \text {. } \\
\text { University of California Davis: PRED, } n=4 ; A Z A, n=1 ; M M F, n=5 \text {. }\end{array}$} \\
\hline
\end{tabular}

therapy with azathioprine or mycophenolate mofetil reduced the incidence of TEAEs, and was associated with a similar combined incidence of death, lung transplantation and respiratory hospitalisation when compared to therapy with prednisone alone.

To our knowledge, this is the first study to specifically explore the outcomes and safety of azathioprine or mycophenolate mofetil in individuals with CHP across multiple tertiary ILD centres in the USA. Our results indicate that when immunosuppressive therapy is necessary, azathioprine and mycophenolate mofetil can be used safely in patients with CHP. These findings are consistent with recent studies by MORISSET et al. [14] that demonstrated no decline in FVC with the use of azathioprine or mycophenolate mofetil therapy in CHP. Similar to autoimmune disorders such as systemic scleroderma-associated ILD in which azathioprine or mycophenolate mofetil are often used as maintenance therapies [15-17], our findings support a possible role for autoimmunity in the disease pathogenesis of CHP. Furthermore, consistent with previous studies in which inhaled substances may trigger the development of autoimmune disease in susceptible individuals [18-21], a subset of patients with CHP may have co-existing autoimmune features [11]. Whether this population would derive the most benefit from B- and T-lymphocyte inhibition with azathioprine or mycophenolate mofetil is unknown, and should be formally addressed in future studies.

Patients who received immunosuppressive therapy with prednisone, regardless of combination with azathioprine or mycophenolate mofetil, had worsened survival than those who did not receive such therapy. This worsened survival is similar to findings from the PANTHER-IPF trial in which patients with idiopathic pulmonary fibrosis (IPF) who received azathioprine in combination with prednisone and $\mathrm{N}$-acetylcysteine had an increased risk of death, hospitalisation and IPF exacerbation [22, 23]. Whether the increased mortality in our CHP cohort who received immunosuppressive therapy was a function of their pre-existing disease severity, their disease stage at clinical presentation, a subgroup of patients with a distinct clinical phenotype or a consequence of immunosuppression with corticosteroids is unknown, and deserves further exploration in larger prospective multicentre trials. Whether antifibrotic therapies recently approved for use in patients with IPF could be beneficial in the treatment of CHP is currently being evaluated in an ongoing clinical trial (www.ClinicalTrials.gov identifier NCT02496182).

Our study supports the use of azathioprine or mycophenolate mofetil for the treatment of CHP when immunosuppressive therapy is necessary. In our cohort, patient tolerance was markedly better with mycophenolate mofetil. Fewer patients initially prescribed mycophenolate mofetil transitioned to azathioprine within the first month than those who were initially treated with azathioprine but transitioned to mycophenolate mofetil. In addition, of those who did take azathioprine or mycophenolate mofetil chronically, mycophenolate mofetil therapy was associated with the least incidence of adverse events overall, when graded by adverse event severity and over time. Mycophenolate mofetil use also decreased the median daily prednisone dosage without worsening FVC decline in patients with CHP treated with prednisone. We were unable to test the effect of corticosteroids in patients receiving azathioprine or mycophenolate mofetil as these DMDs were not initiated as monotherapies. Our findings 
that mycophenolate mofetil therapy may reduce adverse events in patients with fibrotic ILD receiving immunosuppression without worsening lung function are consistent with those in patients with connective tissue disease-associated ILD, in which significantly fewer adverse events were noted in the mycophenolate mofetil arm and the course of FVC over time was similar in patients within both treatment arms [24, 25].

There were several limitations to this investigation. First, as this was a retrospective study, neither causation nor all possible adverse events could be determined; thus, our findings only depict an association between identified outcomes and immunosuppressive therapy. Next, due to the limited sample size of patients with serial PFTs in the azathioprine subgroup, we were unable to perform formal longitudinal analysis of FVC in these patients. Third, concurrent therapy with prednisone and either azathioprine or mycophenolate mofetil in our cohort precluded the ability to evaluate azathioprine and mycophenolate mofetil as monotherapies. Fourth, patients treated with azathioprine or mycophenolate mofetil lacked a standardised reason for initiation of these therapies, as there is currently no standard algorithm for when to start DMDs in CHP. Fifth, we assessed all-cause mortality, as the cause of death was not available in all cases. Finally, several patients referred to our ILD centres had been previously treated by the referring community physician. Although this prior therapy was most often with corticosteroids, exposure to other immunosuppressive agents may have occurred. As we could not ascertain this retrospectively, our findings may have been biased by the influence of these therapies prior to referral.

\section{Conclusions}

Our findings demonstrate that CHP patients who did not receive immunosuppressive therapy had better survival than those who did, and highlight the common clinical practice of treating CHP patients with steroid-sparing immunosuppressive therapy at several tertiary referral centres across the USA. While we showed that the requirement for immunosuppressive therapy was associated with increased mortality, use of mycophenolate mofetil or azathioprine may decrease TEAEs without worsening the decline in lung function or transplant-free survival, when compared to prednisone. Our results suggest that when immunosuppressive therapy is necessary, early transition to mycophenolate mofetil or azathioprine should be considered for patients with chronic, fibrotic hypersensitivity pneumonitis. As the impact of immunosuppressive treatment continues to be investigated, prospective studies are required to determine alternative and targeted first-line therapies for patients with CHP.

\section{Acknowledgements}

These data were presented at the 2016 American Thoracic Society International Conference in San Francisco, CA, USA [26]. A. Adegunsoye, J.M. Oldham, S. Montner, J.H. Chung, A.N. Husain, I. Noth, M.E. Strek and R. Vij conceived and designed the study. All authors acquired, analysed and interpreted the data, drafted the manuscript, and provided important intellectual content. A. Adegunsoye, J.M. Oldham, E.R. Fernández Pérez, M. Hamblin, N. Patel, M. Churpek, S. Montner, J.H. Chung, A.N. Husain, I. Noth, M.E. Strek and R. Vij made substantial contributions to the critical revision of the manuscript. All authors approved the final version of the submitted manuscript.

\section{References}

1 Selman M, Pardo A, King TE Jr. Hypersensitivity pneumonitis: insights in diagnosis and pathobiology. Am J Respir Crit Care Med 2012; 186: 314-324.

2 Fernández Pérez ER, Swigris JJ, Forssén AV, et al. Identifying an inciting antigen is associated with improved survival in patients with chronic hypersensitivity pneumonitis. Chest 2013; 144: 1644-1651.

3 Tateishi T, Ohtani Y, Takemura T, et al. Serial high-resolution computed tomography findings of acute and chronic hypersensitivity pneumonitis induced by avian antigen. J Comput Assist Tomogr 2011; 35: 272-279.

4 Walsh SL, Sverzellati N, Devaraj A, et al. Chronic hypersensitivity pneumonitis: high resolution computed tomography patterns and pulmonary function indices as prognostic determinants. Eur Radiol 2012; 22: 1672-1679.

5 Lacasse Y, Girard M, Cormier Y. Recent advances in hypersensitivity pneumonitis. Chest 2012; 142: 208-217.

6 Lubin $\mathrm{M}$, Chen $\mathrm{H}$, Elicker B, et al. A comparison of health-related quality of life in idiopathic pulmonary fibrosis and chronic hypersensitivity pneumonitis. Chest 2014; 145: 1333-1338.

7 Agache IO, Rogozea L. Management of hypersensivity pneumonitis. Clin Transl Allergy 2013; 3: 5.

8 Hoes JN, Jacobs JW, Verstappen SM, et al. Adverse events of low- to medium-dose oral glucocorticoids in inflammatory diseases: a meta-analysis. Ann Rheum Dis 2009; 68: 1833-1838.

9 Johannson K, Ryerson CJ. Making an accurate diagnosis of chronic hypersensitivity pneumonitis. Can Respir J 2014; 21: 371-370.

10 Lacasse Y, Cormier Y. Hypersensitivity pneumonitis. Orphanet J Rare Dis 2006; 1: 25.

11 Adegunsoye A, Oldham JM, Demchuk C, et al. Predictors of survival in coexistent hypersensitivity pneumonitis with autoimmune features. Respir Med 2016; 114: 53-60.

12 Basch E, Jia X, Heller G, et al. Adverse symptom event reporting by patients vs clinicians: relationships with clinical outcomes. J Natl Cancer Inst 2009; 101: 1624-1632.

13 Dueck AC, Mendoza TR, Mitchell SA, et al. Validity and reliability of the US National Cancer Institute's Patient-Reported Outcomes Version of the Common Terminology Criteria for Adverse Events (PRO-CTCAE). JAMA Oncol 2015; 1: 1051-1059.

14 Morisset J, Johannson KA, Vittinghoff E, et al. Use of mycophenolate mofetil or azathioprine for the management of chronic hypersensitivity pneumonitis. Chest 2017; 151: 619-625. 
15 Bérezné A, Ranque B, Valeyre D, et al. Therapeutic strategy combining intravenous cyclophosphamide followed by oral azathioprine to treat worsening interstitial lung disease associated with systemic sclerosis: a retrospective multicenter open-label study. J Rheumatol 2008; 35: 1064-1072.

16 Paone C, Chiarolanza I, Cuomo G, et al. Twelve-month azathioprine as maintenance therapy in early diffuse systemic sclerosis patients treated for 1-year with low dose cyclophosphamide pulse therapy. Clin Exp Rheumatol 2007; 25: 613-616.

17 Tzouvelekis A, Galanopoulos N, Bouros E, et al. Effect and safety of mycophenolate mofetil or sodium in systemic sclerosis-associated interstitial lung disease: a meta-analysis. Pulm Med 2012; 2012: 143637.

18 Doyle TJ, Dellaripa PF, Batra K, et al. Functional impact of a spectrum of interstitial lung abnormalities in rheumatoid arthritis. Chest 2014; 146: 41-50.

19 Kart L, Sarikaya S, Gurel A, et al. Rheumatoid factor seropositivity and rheumatoid symptoms in coal worker's pneumoconiosis. Clin Rheumatol 2003; 22: 365-366.

20 Rocha MC, Santos LM, Bagatin E, et al. Genetic polymorphisms and surface expression of CTLA-4 and PD-1 on T cells of silica-exposed workers. Int J Hyg Environ Health 2012; 215: 562-569.

21 Webber MP, Moir W, Zeig-Owens R, et al. Nested case-control study of selected systemic autoimmune diseases in World Trade Center rescue/recovery workers. 2015; 67: 1369-1376.

22 Idiopathic Pulmonary Fibrosis Clinical Research Network. Randomized trial of acetylcysteine in idiopathic pulmonary fibrosis. N Engl J Med 2014; 370: 2093-2101.

23 Idiopathic Pulmonary Fibrosis Clinical Research N. Prednisone, azathioprine, and $N$-acetylcysteine for pulmonary fibrosis. N Engl J Med 2012; 366: 1968-1977.

24 Swigris JJ, Olson AL, Fischer A, et al. Mycophenolate mofetil is safe, well tolerated, and preserves lung function in patients with connective tissue disease-related interstitial lung disease. Chest 2006; 130: 30-36.

25 Fischer A, Brown KK, Du Bois RM, et al. Mycophenolate mofetil improves lung function in connective tissue disease-associated interstitial lung disease. J Rheumatol 2013; 40: 640-646.

26 Adegunsoye A, Oldham JM, Chen LW, et al. Outcomes in chronic hypersensitivity pneumonitis treated with immunosuppression. Am J Respir Crit Care Med 2016; 193: A6274. 